\title{
Contribution of Statistical Non-Stationarity in Testing of Existence of Penultimate and Prepenultimate Effects in Stereospecific Polymerization
}

\author{
Riichirô CHûJô \\ Department of Polymer Engineering, Tokyo Institute of Technology, Ookayama 2-12-1, \\ Meguro-ku, Tokyo, Japan.
}

(Received August 29, 1969)

\begin{abstract}
A formula for testing the existence of the penultimate effect in stereospecific polymerization can be improved upon to read$$
\Delta \varepsilon_{P}=-k T \ln \{I S /(i-I)(s-S)\}
$$

by considering the contribution made by statistical non-stationarity in the propagating step. This formula is applied to cases in the polymerization of methyl methacrylate initiated by $n$-butyllithium. The linearity of the plots of $\Delta \varepsilon_{P}$ against polymerization temperature is improved in comparison with plots using the original formula. The formulae for testing the existence of prepenultimate effects are also improved. The measures for estimating penultimate and prepenultimate effects and for the contribution made by statistical non-stationarity are also compared.
\end{abstract}

KEY WORDS Stereospecific Polymerization / Penultimate Effect / Prepenultimate Effect / Statistical Non-Stationarity / Poly(Methyl Methacrylate) /

As a result of the progress made in high resolution nuclear magnetic resonance (NMR) spectroscopy for studying the microstructures of synthetic high polymers, many theories with two parameters have been proposed to interpret the polymerization mechanism in the propagation step. However, such theories are, in general, ambiguous in the way the two parameters are introduced, or in the way they are described, despite the fact that in two-parameter theories each parameter must be able to be defined in definite physical terms and its value able to be adequately determined. The author has derived a formula to clarify whether the penultimate effect, which is a typical concept introduced into two-parameter theories, really does exist or not in stereospecific polymerizations. ${ }^{1}$ The formula is given by

$$
\Delta \varepsilon_{P}=-k T \ln \left(4 I S / H^{2}\right)
$$

where $I, H$ and $S$ denote fractions of isotactic, heterotactic and syndiotactic triads in the polymer, respectively, and $k$ and $T$ are the Boltzmann constant and the polymerization temperature in Kelvin, respectively. The right-hand side of Eq. 1 is composed of observable quantities only, provided that the triad fractions are known from the measurement of the NMR spectrum of the polymer. Therefore, if a linear relation is obtained when the quantities, $\Delta \varepsilon_{P}$ ' $\varepsilon$, at different polymerization temperatures, are plotted against the polymerization temperature, we can say that the penultimate effect exists in the propagation step for obtaining the polymer. As the applications of Eq. 1 to the cationic polymerization of methyl vinyl ethers ${ }^{2}$ and others show, it is known that some of them do not give a linear relation between $\Delta \varepsilon_{P}$ and $T$.

In spite of many successful applications of Eq. 1 to the analysis of stereospecific polymerization, a new and difficult problem presents itself, i.e., the effect of the statistical non-stationarity in polymerization on the quantity under consideration. In other words, the quantity, $\Delta \varepsilon_{P}$, does not always show a linear relation with the polymerization temperature, even if the penultimate effect really does exist, because of the statistical non-stationarity of the system. The purpose of the present paper is to extend Eq. 1 to a form applicable to statistically non-stationary systems. Similar extensions are carried out for the equations $^{3}$ to test the existence of the prepenultimate effect. 


\section{DERIVATION OF THE FORMULAE}

Eq. 1 has been obtained from its original form,

$$
\Delta \varepsilon_{P}=-k T \ln \left(\frac{p_{i i} p_{s s}}{p_{i s} p_{s i}}\right)
$$

with the aid of the conditional probabilities, $p_{\alpha \beta}$ 's, $(\alpha, \beta=i$ or $s)$ of adding a monomer in the form of $\beta$ dyad to a growing end which is in the form of $\alpha$ dyad, under the assumption of statistical stationarity,

$$
p_{i} p_{i s}=p_{s} p_{s i}
$$

where $p_{\alpha}$ 's denote the probabilities of adding a monomer in the form of $\alpha$ dyad to any growing end irrespective of its configuration. In Eqs. 2 and 3, subscripts $i$ and $s$ to $p$ denote isotactic and syndiotactic dyads, respectively.

Where only fractions of triad tacticities are known from the measurement of an NMR spectrum of the polymer, further improvement of Eq. 1 is impossible, because only two independent parameters are given under the normalization condition

$$
I+H+S=1
$$

and two independent parameters are needed to evaluate the right-hand side of Eq. 1. However, if the dyad tacticities are also measurable, the third parameter is available to improve Eq. 1, even if the other normalization condition

$$
i+s=1
$$

is considered. Then, the relations

$$
\left.\begin{array}{rl}
I & =p_{i} p_{i i} \\
H & =p_{i} p_{i s}+p_{s} p_{s i} \\
S & =p_{s} p_{s s} \\
i & =p_{i} \\
s & =p_{s}
\end{array}\right\}
$$

between the fractions of triad and dyad tacticities and the probabilistic parameters lead to the equation

$$
\Delta \varepsilon_{P}=-k T \ln \left\{\frac{I S}{(i-I)(s-S)}\right\}
$$

as an extension of Eq. 1 to the statistically nonstationary case. Of course, Eq. 7 coincides with Eq. 1 in the limit of the statistically stationary case, when we have the relations

$$
\begin{aligned}
& i=\frac{I+H}{2} \\
& s=\frac{S+H}{2}
\end{aligned}
$$

Similarly, the formulae ${ }^{3}$

$$
\begin{aligned}
& \Delta \varepsilon_{I}=-k T \ln \left\{\frac{4(\text { All })(\text { Galacto })}{(\text { Altro })^{2}}\right\} \\
& \Delta \varepsilon_{S}=-k T \ln \left\{\frac{4(\text { Ido })(\text { Manno })}{(\text { Gulco })^{2}}\right\}
\end{aligned}
$$

to clarify the existence of isotactic and syndiotactic prepenultimate effects, respectively, with the aid of the fractions of tetrad tacticities, can be extended to

$$
\begin{aligned}
& \Delta \varepsilon_{I}=-k T \ln \left\{\frac{(\text { Allo })(\text { Galacto })}{(I-\text { Allo })(s-S-\text { Galacto })}\right\} \\
& \Delta \varepsilon_{S}=-k T \ln \left\{\frac{(\text { Ido })(\text { Manno })}{(S-I d o)(i-I-\text { Manno })}\right\}
\end{aligned}
$$

with the aid of the fractions of triad and dyad tacticities as well as those of tetrad ones. In Eqs. 9 and 10, the nomenclatures used for the tetrads are the same as those used by Zambelli et al. ${ }^{4}$

The quantity, $\ln \left(4 I S / H^{2}\right)$, appearing in the right-hand side of Eq. 1 is recommended as a measure of the penultimate effect in symmetric polymerization. ${ }^{5}$ Similarly, a quantity, $\ln (I / S)$, has been used $^{5}$ as a measure of the ultimate effect, as the improved form of $\ln (i / s),{ }^{6}$ and it is known that the penultimate and ultimate effects depend experimentally on each other. In order to discuss such dependence more precisely, a quantity $\ln [I S /\{(i-I)(s-S)\}$ should be adopted as a more general measure of the penultimate effect, as seen in the above discussion. Furthermore, a quantity, $\ln \{(i-I) /(s-S)\}$ can be introduced as the third parameter which describes the effect of statistical non-stationarity.

Similar extensions are possible for the formulae to clarify the existence of the penultimate effects in asymmetric polymerization ${ }^{7}$ and ditactic polymerization, ${ }^{8}$ if a sufficient number of parameters can be measured.

\section{AN EXAMPLE OF APPLICATION}

As an example, Eq. 7 and others are applied to the systems of polymerization of methyl 


\section{R. CHûJô}

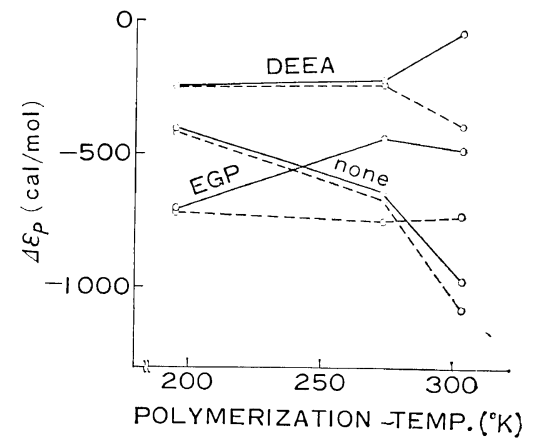

Figure 1. Plots of $\Delta \varepsilon_{P}$ against polymerization temperature for poly(methyl methacrylates) taking into account statistical non-stationarity (broken lines) and disregarding it (solid lines). The designations "none", "DEEA" and "EGP" describe systems without any additive; with 2-diethylaminoethanol and with ethylene glycol monophenyl ether, respectively.

methacrylate in toluene initiated by $n$-butyllithium with some additives. ${ }^{9}$ The values of the mole fractions of triads in the polymers obtained are cited from the original paper in which these values were decided from the intensities of the peaks assigned to the $\alpha-\mathrm{CH}_{3}$ group of the polymers in high resolution NMR spectra. Those of dyads are calculated from the intensities of the peaks assigned to the $-\mathrm{CH}_{2}$ - group of original charts after the resolution of the spectra is enhanced by a digital computer. The method of resolution enhancement is described elsewhere. ${ }^{10}$

Solid lines in Figure 1 are plots of $\Delta \varepsilon_{p}$ 's against polymerization temperatures in these systems with the aid of Eq. 1. The designations "none", "DEEA" and "EGP" inthe figure denote the nature of the additive, i.e., the system without additive, that with 2-diethylaminoethanol and that with ethylene glycol monophenylether, respectively. The linearity of these solid lines is rather poor. A high rate of conversion of monomer to polymer cannot be achieved in every series of polymerization experiments at $303^{\circ} \mathrm{K}$. Thus it cannot be determined at this stage whether the poor linearities of solid lines are due to the contribution of the prepenultimate and further preceding unit effects, or due to the statistical nonstationarity arising from the above-mentioned depression of conversion. Eq. 7 is useful in such cases. Broken lines in Figure 1 are plots similar to the solid ones except for the use of Eq. 7 instead of Eq. 1.

As can be seen from Figure 1, the values of $\Delta \varepsilon_{P}$ 's calculated from Eq. 1 agree with corresponding values from Eq. 7 in lower temperature regions, while these values are in poor agreement in higher temperature regions. It should be noted that the values of $\Delta \varepsilon_{p}$ 's are greatly affected by the accuracy of the experimental data on microtacticities for highly tactic samples because of the amplification of their relative errors. However the agreement in lower temperature regions between $\Delta \varepsilon_{p}$ 's from two kinds of independent data, namely, those of the triad used in Eq. 1 and those of Eq. 7, demonstrate the accuracy of the experimental data in those regions. Furthermore, $\Delta \varepsilon_{P}$ 's in higher temperature regions do not differ greatly from those in lower temperature regions. This tends to demonstrate the accuracy of the data in higher temperature ranges also. As a result, we are able to draw a number of conclusions from Figure 1.

The linearity of the plot with Eq. 7 is somewhat improved at least for the system with EGP. Therefore, it can be concluded that the penultimate effect really exists in the system with EGP in spite of the poor linearity of the curve plotted by Eq. 1, and that there is a considerable amount of statistical non-stationarity in higher temperature regions. The latter may be correlated with the depression of conversion in these regions. If the value of either $p_{i s}$ or $p_{s i}$ is significantly decreased, the rate corresponding to such a small value of the probability may be less than the rate of termination. If this is the case, both statistical non-stationarity and depression of conversion occur in the system.

Plots of $\ln \left(4 I S / H^{2}\right)$ and $\ln \{I S /(i-I)(s-S)\}$ vs. $\ln (I / S)$ (solid lines), $\ln (i / S)$ (broken lines) or $\ln \{(i-I) /(s-S)\}$ (chain lines) for the systems are reproduced in Figures 2 and 3, respectively. Two measures estimating the ultimate effect, $\ln (I / S)$ and $\ln (i / s)$, differ only slightly from each other. A measure for estimating the penultimate effect, $\ln \{I S /(i-I)(s-S)\}$ gives better linearity for the plot against $\ln (I / S)$, etc. compared with the plot of the other one, $\ln \left(4 I S / H^{2}\right)$. Furthermore, a measure for estimating statistical nonstationarity, $\ln \{(i-I) /(s-S)\}$ is also correlated 


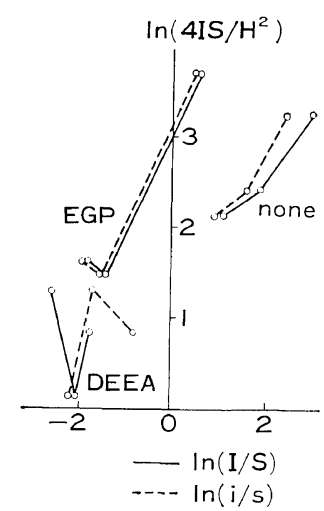

Figure 2. Plots of $\ln \left(4 I S / H^{2}\right)$ vs. $\ln (I / S)$ (solid lines) and $\ln (i / s)$ (broken lines) for poly(methyl methacrylate).

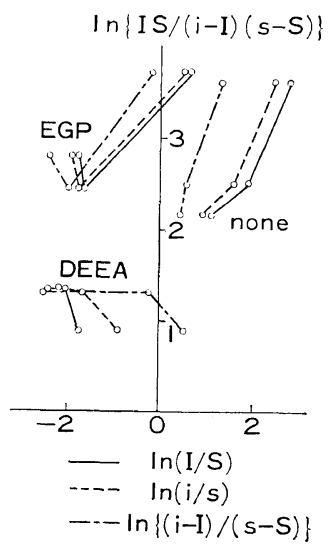

Figure 3. Plots of $\ln \{I S /(i-I)(s-S)\} \quad v s$. $\ln (I / S)$ (solid lines), $\ln (i / s)$ (broken lines) and $\ln \{(i-I)$ $/(s-S)\}$ (chain lines) for poly (methyl methacrylate).

with the ultimate and penultimate effects. Of course, it is not yet known whether these statements can be generally accepted in stereospecific polymerization at all.

\section{CONCLUSION}

The formulae to test the existence of penultimate and prepenultimate effects are extended to Eq. 7 and 10 , respectively, for the statistically nonstationary case. Eq. 7 gives better linearity in the $\Delta \varepsilon_{P} \quad v s$. polymerization temperature plot than does Eq. 1. Therefore if independent information on dyad tacticities is provided, the use of Eq. 7 can be recommended for the study of stereospecific polymerization. Other parameters describing the characteristics of the propagation step in stereospecific polymerization are also discussed and it may be concluded that the use of parameters taking into account statistical non-stationarity is preferable to not considering the effect.

Acknowledgement. The author wishes to express his sincere thanks to Dr. Takida of The Nippon Synthetic Chemical Industry Co., Ltd. for making avaiable the NMR charts used in the preparation of this paper.

\section{REFERENCES}

1. R. Chûjô, J. Phys. Soc. Japan, 21, 2669 (1966).

2. Y. Ohsumi, T. Higashimura, and S. Okamura, J. Polym. Sci., Part A-1, 5, 849 (1967).

3. R. Chûjô, Makromol. Chem., 107, 142 (1967).

4. A. Zambelli, A. L. Segre, M. Farina, and G. Natta, Makromol. Chem., 110, 1 (1967).

5. J. Furukawa, Kobunshi (High Polymers, Japan), 16, 520 (1967).

6. F. A. Bovey, J. Polym. Sci., 46, 59 (1960).

7. R. Chûjô, Repts. Prog. Polym. Phys. Japan, 11, 449 (1968).

8. Y. Ohsumi, T. Higashimura, S. Okamura, R. Chûjô, and T. Kuroda, J. Polym. Sci., Part A-1, 6, 3015 (1968).

9. M. Maruhashi and H. Takita, Makromol. Chem., 124, 172 (1969).

10. R. Chûjô and T. Kuroda, Polymer Journal, 2, 204 (1970). 